\title{
NEIGHBORLY FUNCTIONS
}

\author{
WOODROW W. BLEDSOE
}

1. Introduction. In the following $\rho$ metrizes $S$ and $\rho^{\prime}$ metrizes $S^{\prime}$. We agree ${ }^{1}$ that a function $f$ is neighborly at the point $x$ if and only if for each $\epsilon>0$ there exists ${ }^{2}$ an open sphere $\alpha$ of $S$ such that $\rho(x, y)$ $+\rho^{\prime}(f(x), f(y)) \leqq \epsilon$ whenever $y \in \alpha$. We also agree that a function is neighborly if and only if it is neighborly at each point of $S$. Obviously every continuous function is neighborly.

It is well known that if $g$ is a function on $S$ to $S^{\prime}$ and if $f$ is such a sequence of continuous functions that $\lim _{n \rightarrow \infty} \rho^{\prime}\left(f_{n}(x), g(x)\right)=0$ for each $x$ in $S$, then the points of discontinuity of $g$ form a set of first $\rho$ category. It is the principal purpose of the present note to show that this same conclusion can be drawn when the approximating functions are merely restricted to being neighborly.

2. TheOREM. If $g$ is a function on $S$ to $S^{\prime}$ and $f$ is such a sequence of neighborly functions that $\lim _{n \rightarrow \infty} \rho^{\prime}\left(f_{n}(x), g(x)\right)=0$ for each $x$ in $S$, then the points of discontinuity of $g$ form a set of first $\rho$ category.

Proof. Let $w(x)=\lim \sup _{y \rightarrow x} \rho^{\prime}(g(x), g(y))$ for $x$ in $S$. Since the set of points of discontinuity of $g$ is the set where $w(x)>0$, the desired conclusion is a consequence of the following statement.

Statement. If $n$ is a non-negative integer and $0<\epsilon<\infty$ and $A=S E x\left(w(x) \geqq \epsilon\right.$ and $\rho^{\prime}\left(f_{m}(x), g(x)\right) \leqq \epsilon / 16$ for each integer $\left.m \geqq n\right)$, then $A$ is $\rho$ nondense.

Proof. Suppose

$A$ is dense in some open sphere $\alpha$.

Let $x_{1} \in A \alpha$ and use the neighborliness of $f_{n}$ to secure such an open sphere $\alpha_{1}$ that $\alpha_{1} \subset \alpha$ and

$$
\rho^{\prime}\left(f_{n}\left(x_{1}\right), f_{n}(z)\right) \leqq \epsilon / 16 \quad \text { whenever } z \in \alpha_{1} .
$$

Let $x \in \alpha_{1}$ and choose such an integer $m$ that $m \geqq n$ and

$$
\rho^{\prime}\left(f_{m}(x), g(x)\right) \leqq \epsilon / 16 .
$$

Now use the neighborliness of $f_{m}$ to secure such an open sphere $\alpha_{2}$ that $\alpha_{2} \subset \alpha_{1}$ and

Received by the editors April 4, 1951.

1 The author is indebted to A. P. Morse for several helpful suggestions and for the present concept of a neighborly function.

2 It should be noted that we do not require that $x \in \alpha$. 


$$
\rho^{\prime}\left(f_{m}(x), f_{m}(z)\right) \leqq \epsilon / 16 \quad \text { whenever } z \in \alpha_{2} .
$$

Let $x_{2} \in A \alpha_{2}$. From (3), (4), (2) and the fact that $x_{2} \in A$ and $x_{1} \in A$, it follows that

$$
\begin{aligned}
\rho^{\prime}\left(g(x), g\left(x_{1}\right)\right) \leqq & \rho^{\prime}\left(g(x), f_{m}(x)\right)+\rho^{\prime}\left(f_{m}(x), f_{m}\left(x_{2}\right)\right) \\
& +\rho^{\prime}\left(f_{m}\left(x_{2}\right), g\left(x_{2}\right)\right)+\rho^{\prime}\left(g\left(x_{2}\right), f_{n}\left(x_{2}\right)\right) \\
& +\rho^{\prime}\left(f_{n}\left(x_{2}\right), f_{n}\left(x_{1}\right)\right)+\rho^{\prime}\left(f_{n}\left(x_{1}\right), g\left(x_{1}\right)\right) \\
\leqq & \epsilon / 16+\epsilon / 16+\epsilon / 16+\epsilon / 16+\epsilon / 16+\epsilon / 16 \\
= & 3 \epsilon / 8 .
\end{aligned}
$$

Thus $\rho^{\prime}\left(g(x), g\left(x_{1}\right)\right) \leqq 3 \epsilon / 8$ whenever $x \in \alpha_{1}$. Accordingly $\rho^{\prime}(g(x)$, $g(y)) \leqq 3 \epsilon / 4$ whenever $x \in \alpha_{1}$ and $y \in \alpha_{1}$. Thus $w(x) \leqq 3 \epsilon / 4$ whenever $x \in \alpha_{1}, A \alpha_{1}$ is empty and, in contradiction to (1), $A$ is not dense in $\alpha$. Therefore $A$ is $\rho$ nondense.

3. REMARK. In order to appraise the generality of Theorem 2 we agree that $f$ is neighborly' at the point $x$ if and only if for each $\epsilon>0$ there exists a sphere $\alpha$ of $S$ such that $\rho(x, y)+\rho^{\prime}(f(y), f(z)) \leqq \epsilon$ whenever $y \in \alpha$ and $z \in \alpha$. We further agree that $f$ is neighborly' if and only if it is neighborly' at each point of $S$. Now it is clear that any neighborly function is neighborly'. Moreover the set of points of discontinuity of any neighborly' function is of the first $\rho$ category. However, it is easily seen that the statement resulting from Theorem 2 by replacing "neighborly" by "neighborly" is not a theorem.

4. REMARK. The function $f$ on the real numbers for which

$$
f(x)= \begin{cases}\sin 1 / x & \text { for } x \neq 0 \\ 0 & \text { for } x=0\end{cases}
$$

is neighborly. Also, if for each real $x, f$ is continuous from the right or from the left at $x$, then $f$ is a neighborly function.

It is easy to see that a function on the reals may be neighborly at a point but discontinuous everywhere. Furthermore it is possible to find a neighborly function which is in Baire's second class and yet discontinuous almost everywhere. It is also possible to find a neighborly function which is nonmeasurable relative to every measurable set of positive measure. Such a function is of course discontinuous almost everywhere.

The limit of a sequence of neighborly functions is not necessarily neighborly, but a uniform limit of neighborly functions is neighborly.

University of California, Berkeley 